\title{
Different prognostic effect of CpG island methylation according to sex in colorectal cancer patients treated with adjuvant FOLFOX
}

Dae-Won Lee', Sae-Won Han ${ }^{1,2^{*}}$, Yongjun Cha', Ye Young Rhee ${ }^{3}$, Jeong Mo Bae ${ }^{3}$, Nam-Yun Cho ${ }^{3}$, Kyung-Hun Lee ${ }^{1}$, Tae-Yong Kim', Do-Youn Oh ${ }^{1,2}$, Seock-Ah Im ${ }^{1,2}$, Yung-Jue Bang ${ }^{1,2}$, Seung-Yong Jeong ${ }^{4}$, Kyu Joo Park', Gyeong Hoon Kang ${ }^{3 *}$ and Tae-You Kim ${ }^{1,2,5}$

\begin{abstract}
Background: Profound methylation of $\mathrm{CpG}$ islands constitutes a distinct molecular subtype of colorectal cancer (CRC). The frequencies of methylation in CRC vary according to clinico-pathological characteristics including sex. However, interaction between these characteristics and prognostic influence of methylation status has not been clearly defined. We have investigated the prognostic role of promoter methylation using eight CpG island methylator phenotype (CIMP) markers in 497 stage II or III CRC patients who underwent curative resection followed by adjuvant FOLFOX. Overall survival (OS) and disease-free survival (DFS) were compared between subgroups classified by methylation status, and interactions with clinico-pathological features were analyzed.

Results: CIMP-high ( $\geq 5$ methylated loci) and concurrent methylation in NEUROG1 and CDKN2A (p16) were found in 5.8 and $7.9 \%$ of patients, respectively. Although CIMP-high status was not associated with survival, concurrent methylation in NEUROG1 and CDKN2A (p16) was associated with shorter OS and DFS. Moreover, the prognostic role of the concurrent methylation was different among sex. The negative prognostic impact was only observed in male but not in female (interaction $p$ value $=0.026$ for OS and 0.011 for DFS). In male, the 5 -year OS was $61.6 \%$ in concurrent methylation $(+)$ and $91.7 \%$ in concurrent methylation $(-)(p<0.001)$ whereas it was 95.0 and $92.8 \%$ in female, respectively $(p=0.78)$.

Conclusions: Concurrent methylation in NEUROG1 and CDKN2A is associated with poor survival in CRC treated with adjuvant FOLFOX. Interaction analysis indicates that the prognostic role is different according to sex.
\end{abstract}

Keywords: Colorectal cancer, CpG islands methylator phenotype, Sex, FOLFOX

\section{Background}

Colorectal cancer develops through various types of genetic and epigenetic alterations, and several critical genes and pathways underlying the carcinogenesis have been elucidated. Most notably, at least three distinct pathways have generally been accepted, the chromosomal instability (CIN), microsatellite instability (MSI), and CpG island methylator phenotype (CIMP) pathways. CIMP is characterized by a high frequency of methylation in

\footnotetext{
* Correspondence: saewon1@snu.ac.kr; ghkang@snu.ac.kr

'Department of Internal Medicine, Seoul National University Hospital, 101

Daehang-Ro, Jongno-Gu, Seoul 110-744, South Korea

${ }^{3}$ Department of Pathology, Seoul National University College of Medicine,

101 Daehang-Ro, Jongno-Gu, Seoul 110-744, South Korea

Full list of author information is available at the end of the article
}

numerous promoter $\mathrm{CpG}$ islands. CIMP-positive cancers have distinct features compared to CIN cancers that they are more frequently observed in proximal location, older and female patients, and have poor pathologic differentiation $[1-3]$.

There have been controversies in the prognostic role of CIMP in colorectal cancer patients [3-8]. The inconsistency may be related to CIMP definition using different methylation markers and confounding role of other molecular alterations such as MSI or BRAF mutation. The prognostic implication of CIMP could also be different according to tumor locations [8, 9]. Moreover, methylation status of individual genes may be more important than the number of methylated markers in 
determining prognosis. We have recently reported that concurrent methylation in NEUROG1 and CDKN2A (p16) is associated with higher recurrence in colorectal cancer patients whereas CIMP classification based on the number of methylated markers was not [4]. Importance of individual gene methylation such as CHFR, MGMT, and SHISA3 has also been shown in other studies $[6,10,11]$.

Sex influences clinico-pathological characteristics of colorectal cancer. Male has a higher age-adjusted colorectal cancer incidence and death rate compared to female [12, 13]. The proportion of proximal cancer is higher in female whereas distal colon and rectal cancer is more frequent in male [14]. CIMP also has sexual difference that the frequency is higher in female [1]. The etiology for the sex difference remains uncertain while hormonal factor, dietary factor, and lifestyle factor have been suggested as the cause [15-17].

In the present study, we have analyzed the impact of methylation status on survival in 497 stage III or highrisk stage II colorectal cancer patients treated with adjuvant FOLFOX chemotherapy. We have further investigated whether the prognostic implication is different according to clinico-pathological characteristics including sex.

\section{Result}

\section{Patients' characteristics}

A total of 497 patients were included in the present study. Baseline characteristics are summarized in Table 1. Tumor location was cecum in 18 , ascending colon in 113, transverse in 39, descending in 31, sigmoid in 264, and rectum in 32 patients. Collectively, 169 patients had tumor in proximal (from cecum to transverse colon) location and 328 patients had tumor in distal location. Tumor stage was stage II in 74 patients (IIA in 49, IIB in 21 , and IIC in 4) and stage III in 423 patients (IIIA in 39, IIIB in 273, and IIIC in 111). All stage II patients had high-risk features. Microsatellite instability (MSI-high) was shown in $6.5 \%$ of tumors. According to the inclusion criteria, all patients received at least 6 cycles of chemotherapy and $89.9 \%$ of patients completed planned 12 cycles of chemotherapy.

\section{Methylation status}

Methylation at one or more loci was observed in 181 patients (36.4\%, Table 2). CRABP1 was the most frequently methylated locus, followed by NEUROG1 and CDKN2A (p16). Twenty-nine patients (5.8 \%) had tumors with five or more methylated loci (CIMP-high), 152 patients $(30.6 \%)$ had one to four methylated loci (CIMP-low), and 316 patients (63.6\%) had no methylated locus (CIMP-negative). Patients with CIMP-high tumors were designated the $\operatorname{CIMP}(+)$, and those with CIMP-low or CIMP-negative tumors were designated the CIMP(-). Patients with following characteristics had higher incidence of $\mathrm{CIMP}(+)$ : female sex, proximal tumor location, mucinous adenocarcinoma histology, MSI-high, and BRAF mutation (Table 1). CIMP $(+)$ tumors had a tendency of lower incidence in obese patients (BMI $>25 \mathrm{~kg} / \mathrm{m}^{2}$ for Asian) compared to CIMP(-) $(p=0.096)$. Incidence of $\operatorname{CIMP}(+)$ and individual gene methylation was similar among age.

We previously reported that concurrent methylation in NEUROG1 and CDKN2A (p16) was associated with higher recurrence [4]. Concurrent methylation in NEUROG1 and CDKN2A (p16) was found in 39 patients (7.9\%). Similar to CIMP(+), concurrent methylation had higher incidence in patients with proximal tumor location, mucinous adenocarcinoma histology, MSI-high, and BRAF mutation. Although statistically not significant, concurrent methylation had a tendency of higher incidence in patients with female sex (51.3 vs. $38.4 \%$, $p=0.115$ ). In addition, $\mathrm{N} 2$ stage was higher in patients with concurrent methylation in NEUROG1 and $C D K N 2 A$ ( $p 16$ ) (41.0 vs. $25.5 \%, p=0.036$ ). Incidence of obesity was similar regardless of the concurrent methylation status $(25.6 \%$ in patients with concurrent methylation vs. $35.8 \%$ in patients without concurrent methylation, $p=0.20$ ).

\section{Prognosis according to methylation status}

After a median follow-up duration of 65 months, the 5 -year overall survival (OS) of the entire cohort was $91.1 \%$ and the 3-year disease-free survival (DFS) was $87.2 \%$. There was no significant difference in OS or DFS according to the CIMP status: the 5-year OS was $89.7 \%$ in the CIMP $(+)$ and $91.1 \%$ in the CIMP $(-)(p=$ 0.28 , Fig. 1a) (DFS, Fig. 1b). There was no difference in the pattern of recurrence (local recurrence vs. distant metastasis) according to the CIMP status.

We next evaluated the influence of concurrent methylation in NEUROG1 and CDKN2A (p16) on survival. Concurrent methylation in NEUROG1 and CDKN2A (p16) was associated with poor OS and DFS (Fig. 1c) (DFS, Fig. 1d). The 5-year OS was $78.9 \%$ in patients with concurrent methylation in NEUROG1/CDKN2A (p16) and $92.1 \%$ in patients without concurrent methylation in NEUROG1/CDKN2A $(p<0.001)$. Multivariate analysis using the Cox proportional hazard model revealed that concurrent methylation in NEUROG1 and CDK2NA (p16) was an independent negative prognostic factor for OS (adjusted hazard ratio (HR) for OS 2.89, $95 \%$ confidence interval (CI) 1.45-5.76, $p=0.002$ ) but not for DFS.

\section{Interaction between methylation status and clinico-pathological factors}

We next assessed whether the detrimental effect of concurrent methylation in NEUROG1 and CDKN2A (p16) 
Table 1 Baseline characteristics

\begin{tabular}{|c|c|c|c|c|c|}
\hline & Total & CIMP-negative & CIMP-low & CIMP-high & $p$ value $^{*}$ \\
\hline & $N(\%)$ & $N(\%)$ & $N(\%)$ & $N(\%)$ & \\
\hline Total & $497(100)$ & $316(63.6)$ & $152(30.6)$ & $29(5.8)$ & \\
\hline \multicolumn{6}{|l|}{ Age } \\
\hline$<65$ years & $348(70.0)$ & $222(70.3)$ & $108(71.1)$ & $18(62.1)$ & \multirow[t]{2}{*}{0.34} \\
\hline$\geq 65$ years & $149(30.0)$ & $94(29.7)$ & $44(28.9)$ & $11(37.9)$ & \\
\hline \multicolumn{6}{|l|}{ Sex } \\
\hline Male & 301 (60.6) & 201 (63.6) & $88(57.9)$ & $12(41.4)$ & \multirow[t]{2}{*}{0.029} \\
\hline Female & $196(39.4)$ & $115(36.4)$ & $64(42.1)$ & $17(58.6)$ & \\
\hline \multicolumn{6}{|l|}{ Location } \\
\hline Proximal & $169(34.0)$ & 87 (27.5) & $61(40.1)$ & $21(72.4)$ & \multirow[t]{2}{*}{$<0.001$} \\
\hline Distal & $328(66.0)$ & $229(72.5)$ & 91 (59.9) & $8(27.6)$ & \\
\hline \multicolumn{6}{|l|}{ BMI } \\
\hline$<25 \mathrm{~kg} / \mathrm{m}^{2}$ & $323(65.0)$ & 197 (62.3) & 103 (67.8) & $23(79.9)$ & \multirow[t]{2}{*}{0.096} \\
\hline$\geq 25 \mathrm{~kg} / \mathrm{m}^{2}$ & $174(35.0)$ & $119(37.7)$ & 49 (32.2) & $6(20.7)$ & \\
\hline \multicolumn{6}{|l|}{ T stage } \\
\hline $\mathrm{T} 1-3$ & $424(85.3)$ & $274(86.7)$ & $128(84.2)$ & $22(75.9)$ & \multirow[t]{2}{*}{0.14} \\
\hline $\mathrm{T} 4$ & $73(14.7)$ & $42(13.3)$ & $24(15.8)$ & $7(24.1)$ & \\
\hline \multicolumn{6}{|l|}{ N stage } \\
\hline No-1 & 364 (73.2) & $228(72.2)$ & $116(76.3)$ & $20(69.0)$ & \multirow[t]{2}{*}{0.59} \\
\hline N2 & 133 (26.8) & $88(27.8)$ & $36(23.7)$ & $9(31.0)$ & \\
\hline \multicolumn{6}{|l|}{ Tumor stage } \\
\hline II, high-risk & $74(14.9)$ & $48(15.2)$ & $21(13.8)$ & $5(17.2)$ & \multirow[t]{2}{*}{0.71} \\
\hline III & $423(85.1)$ & $268(84.8)$ & 131 (86.2) & $24(82.8)$ & \\
\hline \multicolumn{6}{|l|}{ Histology } \\
\hline MAC & $25(5.0)$ & $7(2.2)$ & $12(7.9)$ & $6(20.7)$ & \multirow[t]{2}{*}{$<0.001$} \\
\hline Non-MAC & $472(95.0)$ & 309 (97.8) & $140(92,1)$ & $23(79.3)$ & \\
\hline \multicolumn{6}{|c|}{ Microsatellite status $(N=495)$} \\
\hline MSS/MSI-L & $463(93.5)$ & $306(97.5)$ & $138(90.8)$ & $19(65.5)$ & \multirow[t]{2}{*}{$<0.001$} \\
\hline MSI-H & $32(6.5)$ & $8(2.5)$ & $14(9.2)$ & $10(34.5)$ & \\
\hline \multicolumn{6}{|c|}{ KRAS mutation $(N=383)$} \\
\hline Wild type & $280(73.1)$ & $185(76.4)$ & $77(64.2)$ & $18(85.7)$ & \multirow[t]{2}{*}{0.18} \\
\hline Mutation & $103(26.9)$ & $57(23.6)$ & $43(35.8)$ & $3(14.3)$ & \\
\hline \multicolumn{6}{|c|}{ BRAF mutation $(N=423)$} \\
\hline Wild type & $407(96.2)$ & $270(99.3)$ & $122(93.8)$ & $15(71.4)$ & \multirow[t]{2}{*}{$<0.001$} \\
\hline Mutation & $16(3.8)$ & $2(0.7)$ & $8(6.2)$ & $6(28.6)$ & \\
\hline
\end{tabular}

$\mathrm{N}$ number, MAC mucinous adenocarcinoma, MSS microsatellite stable, MSI-L microsatellite instability-low, MSI- $H$ microsatellite instability-high

${ }^{*} p$ values comparing CIMP-high vs. CIMP-low/CIMP-negative by chi-square test

was different according to clinico-pathological factors, including sex (Fig. 2). The prognostic role of concurrent methylation in NEUROG1 and CDKN2A (p16) was different among sex (interaction $p$ value for $\mathrm{OS}=0.026$, for $\mathrm{DFS}=0.011)$. It was associated with significantly worse OS and DFS in men (Fig. 3a) (DFS, Fig. 3c). However, there was no prognostic role of concurrent methylation in women (Fig. 3b) (DFS, Fig. 3d). In the multivariate analysis, the poor prognosis associated with concurrent methylation in NEUROG1 and CDKN2A (p16) in male was independent of other clinico-pathologic prognostic factors (adjusted HR for OS 5.23, $95 \%$ CI 2.45-11.17, $p<0.001$ ) (adjusted HR for DFS 3.66, 95 \% CI 1.82-7.36, $p<0.001$ ) (Table 3). Other clinico-pathological factors, including tumor location, did not affect the prognostic role of concurrent methylation in NEUROG1 and 
Table 2 Summary of methylation status

\begin{tabular}{llll}
\hline $\begin{array}{l}\text { Number of } \\
\text { methylated loci }\end{array}$ & $\begin{array}{l}\text { Number of } \\
\text { patients (\%) }\end{array}$ & $\begin{array}{l}\text { Methylation } \\
\text { locus }\end{array}$ & $\begin{array}{l}\text { Number of patients } \\
\text { with methylation (\%) }\end{array}$ \\
\hline 0 & $316(63.6)$ & CACNA1G & $41(8.2)$ \\
1 & $94(18.9)$ & CRABP1 & $98(19.7)$ \\
2 & $37(7.4)$ & IGF2 & $30(6.0)$ \\
3 & $13(2.6)$ & MLH1 & $18(3.6)$ \\
4 & $8(1.6)$ & NEUROG1 & $87(17.5)$ \\
5 & $13(2.6)$ & CDKN2A & $85(17.1)$ \\
6 & & (p16) & \\
7 & $9(1.8)$ & RUNX3 & $29(5.8)$ \\
8 & $4(0.8)$ & SOCS1 & $22(4.4)$ \\
\hline
\end{tabular}

CDKN2A (p16). Due to the limited number of patients, we could not sub-analyze patients according to $B R A F$ mutation or MSI status.

\section{Discussion}

In the present study, we have investigated the impact of promoter methylation on treatment outcome of colorectal cancer patients receiving adjuvant FOLFOX chemotherapy. As all patients in our cohort were Korean, our data shows relatively low incidence of MSI-high (MSI-H) and CIMP-high. Previous studies using standardized methodologies have repeatedly shown that the Western shows higher incidence of MSI-H and CIMPhigh compared to the Eastern [18]. We observed that CIMP-high is not associated with survival, but concurrent methylation in NEUROG1 and CDKN2A (p16) has deleterious effect in terms of OS and DFS. In addition, the prognostic role of concurrent methylation in NEUROG1 and $C D K N 2 A$ was different among sex; it was a negative prognostic factor in men but not in women (interaction $p$ value of 0.026 for OS and 0.011 for DFS).

Although the prognostic role of CIMP has been extensively investigated in colorectal cancer patients, previous studies have yielded inconsistent results. In the study by Min et al., CIMP-high was a positive prognostic factor in stage II or III colorectal cancer patients treated with fluoropyrimidine-based adjuvant chemotherapy [7]. However, CIMP-high was a negative prognostic factor in stage III, proximal colon cancer patients [8]. These inconsistent results may have attributed from the heterogeneous cohort of patients included in the study, different CIMP-high definitions among studies and confounding role of other molecular characteristics (MSI-high, KRAS
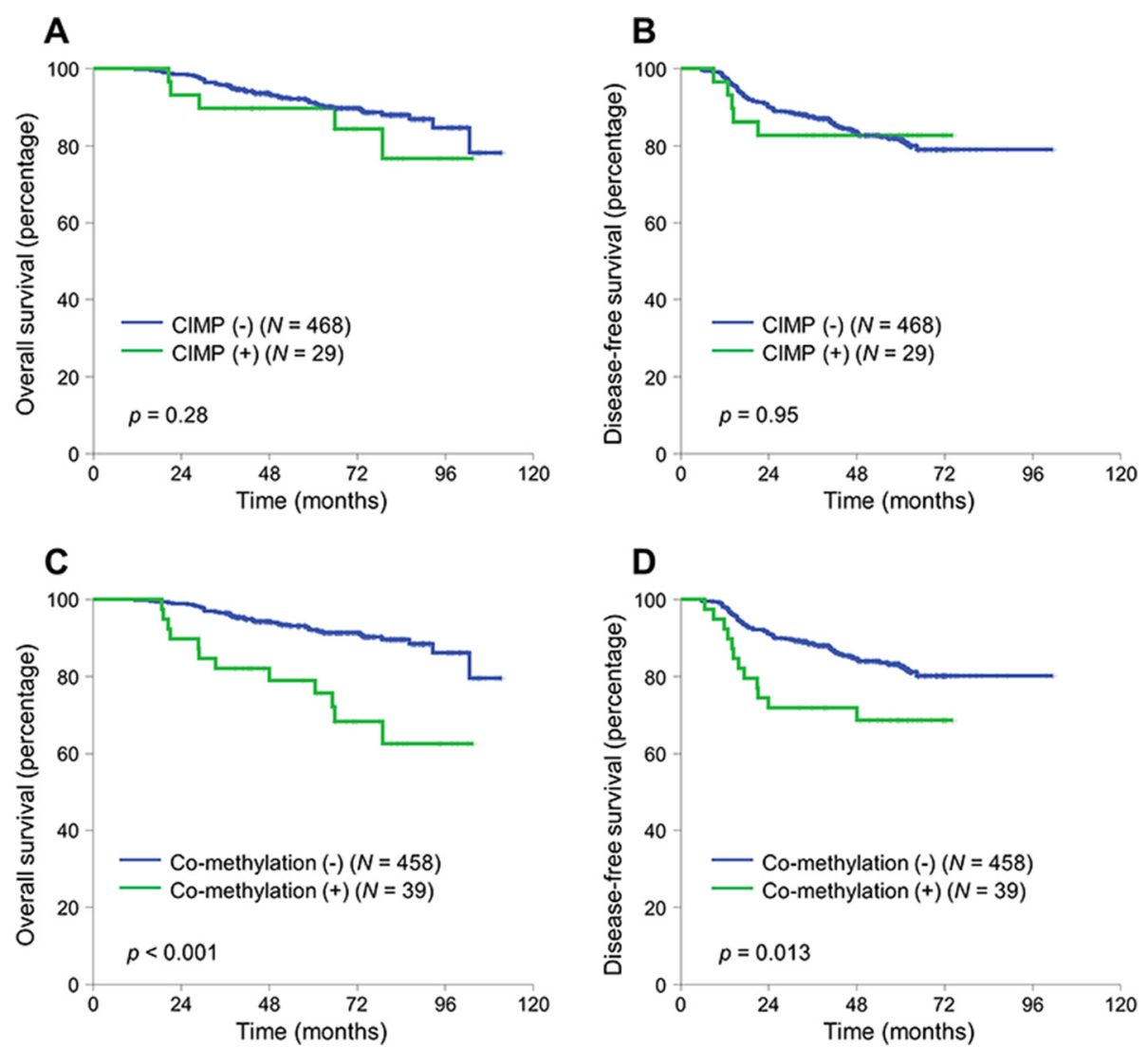

Fig. 1 Kaplan-Meier curves of overall survival and disease-free survival according to CIMP status $(\mathbf{a}, \mathbf{b})$ and concurrent methylation of NEUROG1/CDKN2A (p16) (c, d). Co-methylation concurrent methylation, $N$ number 


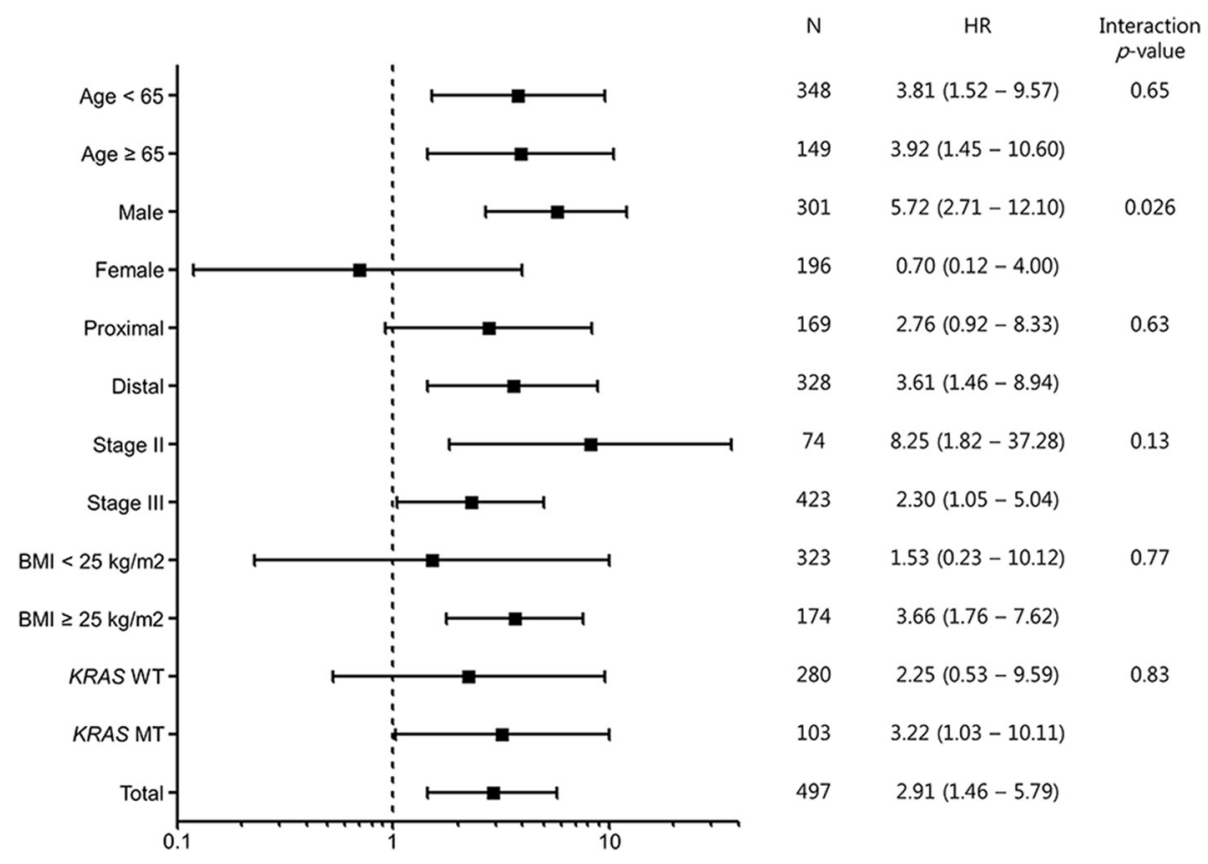

Fig. 2 Forest plot demonstrating the risk of death by concurrent methylation (+) compared to concurrent methylation (-) stratified by clinico-pathological factors. All hazard ratios were adjusted by histology, angiolymphatic invasion, and perineural invasion. $N$ number, HR hazard ratio, Cl confidence interval, WT wild type, MT mutation type

mutation, and $B R A F$ mutation). In addition, there are evidences that each methylation locus differs in their association with survival and clinico-pathological characteristics. Concurrent promoter methylation in NEUROG1 and CDKN2A (p16) was associated with poor DFS in stages II and III colorectal cancer patients, and CHFR promoter methylation indicated poor prognosis in stage II colorectal cancer patients $[4,6]$. In contrast, considerable proportion of MSI-H in sporadic colorectal cancer results from the loss of $M L H 1$ expression by methylation of its promoter, and it is known that MSI-H is associated with better prognosis $[19,20]$. In our study, CIMP-high was not associated with survival but concurrent methylation in NEUROG1 and CDKN2A (p16) was associated with poor survival. NEUROG1 is a transcription factor involved in neuronal development and differentiation, and CDKN2A (p16) is a tumor suppressor that inhibits cyclin-dependent kinases CDK4 and CDK6 [21, 22]. Although the functional role of promoter methylation in NEUROG1 and CDKN2A (p16) is inconclusive, $C D K N 2 A$ (p16) promoter methylation was associated with poor survival in stages II and III colorectal cancer patients who received adjuvant fluoropyrimidinebased chemotherapy [23]. Classifying CIMP according to the number of methylated loci may be useful for grouping patients with similar clinico-pathological characteristics; however, each promoter methylation may have different prognostic roles, and thus, identifying each methylation locus may be useful in the practice.
There are sex differences in colorectal cancer incidence, death rate, and clinico-pathological characteristics [24]. Female colorectal cancer patients tend to have CIMP-high and proximal tumor location compared to male patients. To our knowledge, no study has revealed the interaction between sex and prognostic impact of promoter methylation yet. In this study, we found that the prognostic role of concurrent methylation in $N E U$ ROG1 and CDKN2A (p16) was influenced by sex. It was associated with poor prognosis only in male patients. Previous studies have shown that a negative prognostic role of obesity has sex-related differences $[15,25]$. However, we could not find association between methylation status and obesity. Because of the retrospective nature of this study, we could not examine other potential mechanism underlying the sex differences including hormonal factor, dietary factor, and lifestyle factor. Future prospective cohort study may provide answers to the differences.

Other limitation of this study is that only patients treated with adjuvant FOLFOX were included. Therefore, we cannot answer whether the poor prognosis of patients with concurrent methylation in NEUROG1 and CDKN2A (p16) is due to its innate biology or its resistance to adjuvant chemotherapy regimen. However, the major strength of the study is that the study cohort was homogenous, that all patients underwent surgery at a high-volume center and received the same adjuvant FOLFOX chemotherapy, which is the current standard care in patients with stage 

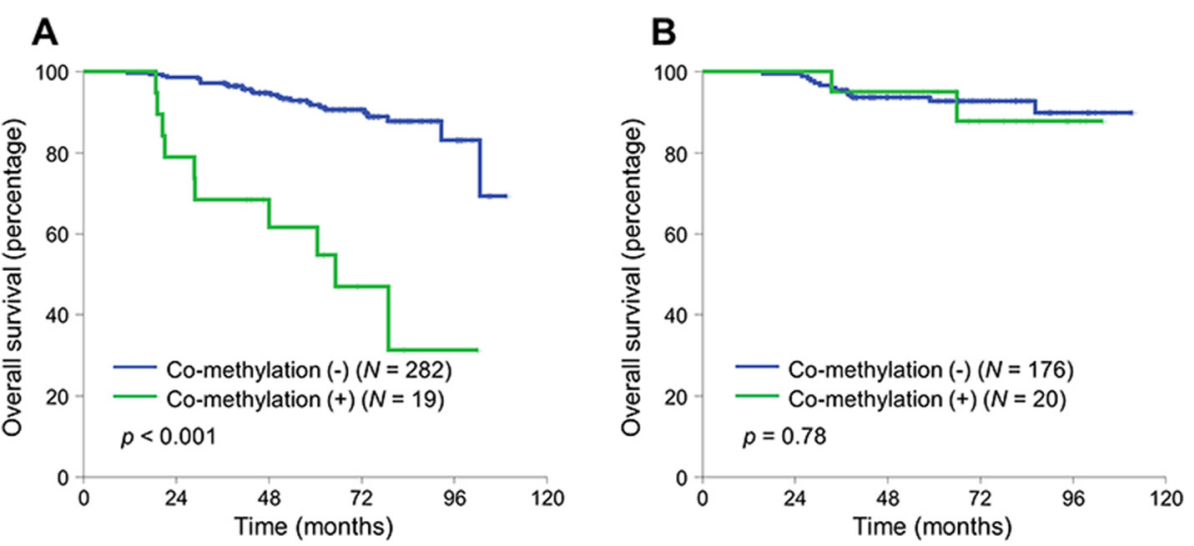

C

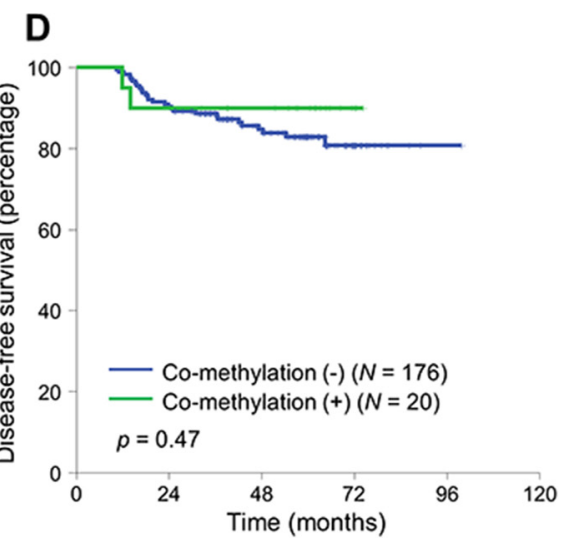

Fig. 3 Kaplan-Meier curves of overall survival (OS) and disease-free survival (DFS) according to concurrent methylation of NEUROG1/CDKN2A (p16) stratified by sex. (a) Male: OS. (b) Female: OS. (c) Male: DFS. (d) Female: DFS. Co-methylation concurrent methylation, $N$ number

III colorectal cancer [26]. Our findings need further validation in an independent cohort of patients.

\section{Conclusions}

While CIMP is well known for its role in colon cancer tumorigenesis, the prognostic role of CIMP has not been well defined. In this study, CIMP-high did not have a prognostic role; however, concurrent methylation in NEUROG1 and CDKN2A (p16) was independently associated with poor survival in colorectal cancer patients treated with adjuvant FOLFOX. In addition, the prognostic role of concurrent methylation in NEUROG1 and CDKN2A (p16) was influenced by sex. A negative prognostic role of promoter methylation was shown in men

Table 3 Multivariate analysis of overall survival and disease-free survival among male patients $(N=301)$

\begin{tabular}{|c|c|c|c|c|c|}
\hline & & \multicolumn{2}{|l|}{ Overall survival } & \multicolumn{2}{|l|}{ Disease-free survival } \\
\hline & & Adjusted HR (95 \% Cl) & $p$ value & Adjusted HR (95 \% Cl) & $p$ value \\
\hline \multirow[t]{2}{*}{ Concurrent methylation } & Present & $5.23(2.45-11.17)$ & $<0.001$ & $3.66(1.82-7.36)$ & $<0.001$ \\
\hline & Not present & 1 & & 1 & \\
\hline \multirow[t]{2}{*}{ Angiolymphatic invasion } & Present & $4.41(1.97-9.85)$ & $<0.001$ & $2.33(1.30-4.18)$ & 0.005 \\
\hline & Not present & 1 & & 1 & \\
\hline Age (continuous variable) & & $1.032(0.99-1.07)$ & 0.11 & & \\
\hline \multirow[t]{2}{*}{ Histology } & MAC & $3.47(0.97-12.41)$ & 0.056 & & \\
\hline & Non-MAC & 1 & & & \\
\hline \multirow[t]{2}{*}{ Perineural invasion } & Present & & & $2.20(1.26-3.85)$ & 0.006 \\
\hline & Not present & & & 1 & \\
\hline
\end{tabular}


but not in women. Elucidating the underlying mechanism that results in sex difference is warranted in the future.

\section{Methods}

\section{Patients and adjuvant chemotherapy}

This study included 497 pathologically proven stage III or high-risk stage II colorectal cancer patients who received curative surgery followed by adjuvant FOLFOX chemotherapy at Seoul National University Hospital (SNUH; Seoul, Korea) between April 2005 and December 2011. Main inclusion criteria for the retrospective patient selection were age over 18 , adenocarcinoma histology, stage III or high-risk stage II, complete resection of the tumor with negative margin, completion of at least 6 cycles of adjuvant FOLFOX chemotherapy. High-risk stage II was defined if the patient had any of the following: T4 lesion, obstruction or perforation, lymphovascular invasion, perineural invasion, or poorly differentiated histology [27]. Patients with upper rectal cancer were included if the patient did not receive preor post-operative radiation. Patients were excluded if they met the following criteria: previous chemotherapy for colorectal cancer (CRC), previous radiotherapy for CRC, signet ring cell histology, distant metastasis, and history of other malignancy within 5 years. None of the patients received anti-EGFR or anti-VEGF treatment adjunct to FOLFOX. Patient received FOLFOX chemotherapy as either FOLFOX-4 (288 patients) or modified FOLFOX-6 (209 patients) regimen [28]. Adjuvant chemotherapy was planned for a total of 12 cycles.

Patients were assessed every 2 weeks during chemotherapy treatment and then at least every 6 months for 5 years. The post chemotherapy period assessment included a medical history taking, physical examination, measurement of the carcinoembryonic antigen level, chest computed tomography, and abdominal computed tomography. The diagnosis of recurrence was made on the basis of imaging and, if necessary, biopsy.

Eligible patients were identified from electronic database, and chart review was performed using the electronic medical record system of SNUH. The study protocol was reviewed and approved by the institutional review board of SNUH.

\section{Molecular pathologic analysis}

Analysis of DNA methylation and microsatellites was performed as previously described [4, 29]. All patients $(N=322)$ included in the previous report were included in the present study [4]. DNA methylation analysis was re-performed for these patients concurrently with the new patients $(N=175)$. In brief, tumor tissue slides were reviewed and areas of high tumor cell density $\left(\sim 1 \mathrm{~cm}^{2}\right)$ were marked and dissected with a knife blade. Non- neoplastic colon mucosa tissues were also dissected. The dissected tumor tissues were collected into a microtube containing tissue lysis buffer and proteinase K. Manual microdissection was performed to enrich tumor cell DNA proportion in the sample DNA, because the results of quantitative MethyLight analysis may be influenced by high proportions of contaminating normal cells. After sodium bisulfite conversion of DNA using the EZ DNA methylation kit (Zymo Research, Orange, CA, USA), the methylation status was quantified using MethyLight assay in the following eight CIMP markers: CACNA1G, CDKN2A (p16), CRABP1, IGF2, MLH1, NEUROG1, RUNX3, and SOCS1. The primer sequences and polymerase chain reaction (PCR) conditions have been described previously $[29,30]$. M.SssI-treated genomic DNA was used as a reference sample. Percentage of methylated reference (PMR) at a particular locus was calculated by dividing the GENE/ALU ratio of a patient sample by the GENE/ALU ratio of the M.SssI-treated human genomic DNA sample and multiplying by 100 . MethyLight assay was repeated in triplicate, and of the three measured values, the median was regarded as a representative value of methylation level of each marker. A CpG island locus with PMR $>4$ was considered to be methylated $[4,29,31]$. CIMP status was defined according to the number of methylated markers: CIMP-high (methylation at $\geq 5$ markers), CIMP-low (1-4 markers), or CIMP-negative (0 marker) [4, 29, 31].

The microsatellite status of each tumor was determined by evaluating the five microsatellite markers (D2S123, D5S346, D17S250, BAT25, and BAT26). Either forward or reverse primer for each marker was labeled with fluorescence, and PCR products were electrophoresed and analyzed. We classified MSI status as follows: MSI-high (MSI-H; instability at two or more microsatellite markers), MSI-low (MSI-L; instability at one marker), or microsatellite stable (MSS) (no instability). Only MSI-H was regarded as having MSI, and MSI-L was grouped with MSS [4, 32].

Analysis of KRAS and BRAF mutation was performed as previously described [32]. DNA was extracted from paraffin-embedded tissue, and KRAS mutation (codon 12 and 13 of exon 2) was analyzed by using hemi-nested PCR method followed by direct sequencing. BRAF mutations at codon 600 (V600E) were analyzed by using a real-time PCR-based allelic discrimination method [32].

\section{Statistical analysis}

The primary objective of this study was to investigate the effect of promoter methylation status on the treatment outcome (OS and DFS) and their association with sex in colorectal cancer patients treated with adjuvant FOLFOX chemotherapy. The clinical database was last updated in October 2014. DFS was calculated from the 
date of operation to the first date of documented recurrence or death. Data from patients who were free of recurrence were censored at the date of the last follow-up visit for DFS. In the analysis of OS, death from any cause was the primary end point. Categorical variables were compared using the chi-square test. OS and DFS were calculated using the Kaplan-Meier method, and comparisons were made using the log-rank tests. HR was calculated using the Cox proportional hazard model, and baseline characteristics were adjusted by using backward stepwise model including covariates which have the prognostic role: age (continuous variable), sex, stage (II vs. III), histology (mucinous adenocarcinoma vs. others), tumor location (proximal vs. distal), angiolymphatic invasion, venous invasion, perineural invasion, and MSI status. Two-sided $p$ values of less than 0.05 were considered statistically significant. Statistical analysis was performed with SPSS software for Windows, version 18.0 (SPSS, Chicago, IL, USA).

\section{Competing interest}

The authors declare that they have no competing interests.

\section{Authors' contributions}

SWH, GHK and DWL were involved in the study concept and design. SWH, DWL, YC, YYR, JMB, NYC, KHL, TYK, DYO, SAI, YJB, SYJ, KJP, GHK, and TYK involved in the data acquisition. The analysis and interpretation of data was done by SWH and DWL. The manuscript writing was made by SWH and DWL. The manuscript review and final approval were done by SWH, DWL, YC, YYR, JMB, NYC, KHL, TYK, DYO, SAI, YJB, SYJ, KJP, GHK, and TYK. All authors read and approved the final manuscript.

\section{Acknowledgements}

This research was supported by grants from the Korea Health Technology R\&D Project (HI14C1277) through the Korea Health Industry Development Institute (KHIDI), funded by the Ministry of Health \& Welfare; the NRF grant funded by the Korea government (MSIP) (2011-0030049); and the Converging Research Center Program funded by the Ministry of Science, ICT and Future Planning (2014M3C1A8048802).

\section{Author details \\ ${ }^{1}$ Department of Internal Medicine, Seoul National University Hospital, 101 Daehang-Ro, Jongno-Gu, Seoul 110-744, South Korea. ${ }^{2}$ Cancer Research Institute, Seoul National University College of Medicine, Seoul, Korea. ${ }^{3}$ Department of Pathology, Seoul National University College of Medicine, 101 Daehang-Ro, Jongno-Gu, Seoul 110-744, South Korea. ${ }^{4}$ Department of Surgery, Seoul National University Hospital, Seoul, Korea. ${ }^{5}$ Department of Molecular Medicine and Biopharmaceutical Sciences, Graduate School of Convergence Science and Technology, Seoul National University, Seoul, Korea.}

Received: 28 April 2015 Accepted: 1 July 2015

Published online: 09 July 2015

\section{References}

1. Issa JP. CpG island methylator phenotype in cancer. Nat Rev Cancer. 2004;4(12):988-93.

2. Barault L, Charon-Barra C, Jooste V, de la Vega MF, Martin L, Roignot $P$, et al. Hypermethylator phenotype in sporadic colon cancer: study on a population-based series of 582 cases. Cancer Res. 2008;68(20):8541-6.

3. Ogino S, Nosho K, Kirkner GJ, Kawasaki T, Meyerhardt JA, Loda M, et al. CpG island methylator phenotype, microsatellite instability, BRAF mutation and clinical outcome in colon cancer. Gut. 2009;58(1):90-6.
4. Han SW, Lee HJ, Bae JM, Cho NY, Lee KH, Kim TY, et al. Methylation and microsatellite status and recurrence following adjuvant FOLFOX in colorectal cancer. Int J Cancer. 2013;132(9):2209-16.

5. Shiovitz S, Bertagnolli MM, Renfro LA, Nam E, Foster NR, Dzieciatkowski S, et al. CpG island methylator phenotype is associated with response to adjuvant irinotecan-based therapy for stage III colon cancer. Gastroenterology. 2014;147(3):637-45.

6. Cleven AH, Derks S, Draht MX, Smits KM, Melotte V, Van Neste $L$, et al. CHFR promoter methylation indicates poor prognosis in stage II microsatellite stable colorectal cancer. Clin Cancer Res. 2014;20(12):3261-71.

7. Min BH, Bae JM, Lee EJ, Yu HS, Kim YH, Chang DK, et al. The CpG island methylator phenotype may confer a survival benefit in patients with stage ॥ or III colorectal carcinomas receiving fluoropyrimidine-based adjuvant chemotherapy. BMC Cancer. 2011;11:344.

8. Ahn JB, Chung WB, Maeda O, Shin SJ, Kim HS, Chung HC, et al. DNA methylation predicts recurrence from resected stage III proximal colon cancer. Cancer. 2011;117(9):1847-54

9. Bae JM, Kim JH, Cho NY, Kim TY, Kang GH. Prognostic implication of the CpG island methylator phenotype in colorectal cancers depends on tumour location. Br J Cancer. 2013;109(4):1004-12.

10. Li Y, Lyu Z, Zhao L, Cheng H, Zhu D, Gao Y, et al. Prognostic value of MGMT methylation in colorectal cancer: a meta-analysis and literature review. Tumour Biol. 2015;36(3):1595-601.

11. Tsai MH, Chen WC, Yu SL, Chen CC, Jao TM, Huang CY, et al. DNA Hypermethylation of SHISA3 in colorectal cancer: an independent predictor of poor prognosis. Ann Surg Oncol. 2015. [Epub ahead on print].

12. Ferlay J, Steliarova-Foucher E, Lortet-Tieulent J, Rosso S, Coebergh JW, Comber $\mathrm{H}$, et al. Cancer incidence and mortality patterns in Europe: estimates for 40 countries in 2012. Eur J Cancer. 2013;49(6):1374-403.

13. Siegel R, Desantis C, Jemal A. Colorectal cancer statistics, 2014. CA Cancer J Clin. 2014;64(2):104-17.

14. Nelson RL, Dollear T, Freels S, Persky V. The relation of age, race, and gender to the subsite location of colorectal carcinoma. Cancer. 1997;80(2):193-7.

15. Sinicrope FA, Foster NR, Sargent DJ, O'Connell MJ, Rankin C. Obesity is an independent prognostic variable in colon cancer survivors. Clin Cancer Res. 2010;16(6):1884-93.

16. Folkerd EJ, Dowsett M. Influence of sex hormones on cancer progression. J Clin Oncol. 2010;28(26):4038-44.

17. Nguyen SP, Bent S, Chen YH, Terdiman JP. Gender as a risk factor for advanced neoplasia and colorectal cancer: a systematic review and meta-analysis. Clin Gastroenterol Hepatol. 2009;7(6):676-81. e1-3.

18. Kim JH, Kang GH. Molecular and prognostic heterogeneity of microsatelliteunstable colorectal cancer. World J Gastroenterol. 2014;20(15):4230-43.

19. Boland $C R$, Goel A. Microsatellite instability in colorectal cancer. Gastroenterology. 2010;138(6):2073-87. 6.

20. Guastadisegni C, Colafranceschi M, Ottini L, Dogliotti E. Microsatellite instability as a marker of prognosis and response to therapy: a meta-analysis of colorectal cancer survival data. Eur J Cancer. 2010;46(15):2788-98.

21. Sun Y, Nadal-Vicens M, Misono S, Lin MZ, Zubiaga A, Hua X, et al. Neurogenin promotes neurogenesis and inhibits glial differentiation by independent mechanisms. Cell. 2001;104(3):365-76.

22. Gil J, Peters G. Regulation of the INK4b-ARF-INK4a tumour suppressor locus: all for one or one for all. Nat Rev Mol Cell Biol. 2006;7(9):667-77.

23. Kim JC, Choi JS, Roh SA, Cho DH, Kim TW, Kim YS. Promoter methylation of specific genes is associated with the phenotype and progression of colorectal adenocarcinomas. Ann Surg Oncol. 2010;17(7):1767-76.

24. Koo JH, Leong RW. Sex differences in epidemiological, clinical and pathological characteristics of colorectal cancer. J Gastroenterol Hepatol. 2010;25(1):33-42

25. Meyerhardt JA, Catalano PJ, Haller DG, Mayer RJ, Benson 3rd AB, Macdonald JS, et al. Influence of body mass index on outcomes and treatment-related toxicity in patients with colon carcinoma. Cancer. 2003;98(3):484-95

26. Andre T, Boni C, Navarro M, Tabernero J, Hickish T, Topham C, et al. Improved overall survival with oxaliplatin, fluorouracil, and leucovorin as adjuvant treatment in stage II or III colon cancer in the MOSAIC trial. J Clin Oncol. 2009;27(19):3109-16

27. Schmoll HJ, Van Cutsem E, Stein A, Valentini V, Glimelius B, Haustermans K, et al. ESMO Consensus Guidelines for management of patients with colon and rectal cancer. a personalized approach to clinical decision making. Ann Oncol. 2012;23(10):2479-516. 
28. Lee DW, Han SW, Lee HJ, Rhee YY, Bae JM, Cho NY, et al. Prognostic implication of mucinous histology in colorectal cancer patients treated with adjuvant FOLFOX chemotherapy. Br J Cancer. 2013;108(10):1978-84.

29. Kim JH, Shin SH, Kwon HJ, Cho NY, Kang GH. Prognostic implications of $\mathrm{CpG}$ island hypermethylator phenotype in colorectal cancers. Virchows Arch. 2009;455(6):485-94.

30. Weisenberger DJ, Siegmund KD, Campan M, Young J, Long TI, Faasse MA, et al. CpG island methylator phenotype underlies sporadic microsatellite instability and is tightly associated with BRAF mutation in colorectal cancer. Nat Genet. 2006;38(7):787-93.

31. Ogino S, Kawasaki T, Brahmandam M, Cantor M, Kirkner GJ, Spiegelman D, et al. Precision and performance characteristics of bisulfite conversion and real-time PCR (MethyLight) for quantitative DNA methylation analysis. J Mol Diagn. 2006;8(2):209-17.

32. Lee DW, Kim KJ, Han SW, Lee HJ, Rhee YY, Bae JM, et al. KRAS mutation is associated with worse prognosis in stage III or high-risk stage II colon cancer patients treated with adjuvant FOLFOX. Ann Surg Oncol. 2015;22(1):187-94

\section{Submit your next manuscript to BioMed Central and take full advantage of:}

- Convenient online submission

- Thorough peer review

- No space constraints or color figure charges

- Immediate publication on acceptance

- Inclusion in PubMed, CAS, Scopus and Google Scholar

- Research which is freely available for redistribution 